\title{
Stress effects on the performance of optical waveguides
}

\author{
M. Huang * \\ Mechanical and Aerospace Engineering Department and Princeton Materials Institute, Princeton University, Princeton, NJ 08544, USA
}

Received 5 August 2002; received in revised form 5 December 2002

\begin{abstract}
Stresses can cause anisotropic and inhomogeneous distribution of the refractive index. Their effects on the performance of optical waveguides have been observed in photoelectric devices. In this paper, the photo-elastic relation and wave equations for inhomogeneous and anisotropic waveguides are reviewed. The effective refractive indexes and mode shapes of planar waveguides under different stress states are obtained analytically. It is found that stress can affect the optical performance; different stress states play different roles: high stress value can change the cutoff thickness, which may induce multimode; in-plane stress causes birefringence, which may induce polarization shift and polarization dependent loss; stress concentration can change the mode shape, which may induced large transition loss; and pure shear stress has little effects on the effective refractive index.
\end{abstract}

(c) 2002 Elsevier Science Ltd. All rights reserved.

Keywords: Stress; Photo-elastic; Waveguide; Birefringence; Loss; Multimode

\section{Introduction}

Optical waveguides are basic components in many optical systems, such as arrayed waveguide gratings (AWGs), lasers and tunable filters. The function of waveguide is to direct the light to the desired location. In modern optical systems, more and more components are integrated (or hybrid-packaged) in a single chip, where optical waveguides serve as interconnects for photonic components. Due to fabrication and packaging, stresses cannot be avoided in these complicated structures with many diverse materials. These stresses can change the refractive indexes of the waveguide by the acousto-optic (elasto-optic or photoelastic) effect (Xu and Stroud, 1992; Sapriel, 1979), which may affect the optical performance. Fig. 1 shows the finite element simulation results of the stress distribution in a ridge $\mathrm{Si}$ waveguide core. This core is covered by silica cladding layer and $\mathrm{Al}$ interconnect layer, which are not shown in this figure. These layers are fabricated at high temperatures (e.g., $300{ }^{\circ} \mathrm{C}$ for $\mathrm{Al}$ interconnect and $1000{ }^{\circ} \mathrm{C}$ for silica cladding), and cooled down to room temperature. The stresses shown in Fig. 1 are due to the thermal mismatch of different layers. Because of the sharp corners and the shape of the waveguide, the stresses are inhomogeneous and

\footnotetext{
${ }^{*}$ Tel.: +1-609-258-0907; fax: +1-609-258-5877.

E-mail address: minhuang@alumni.princeton.edu (M. Huang).
} 

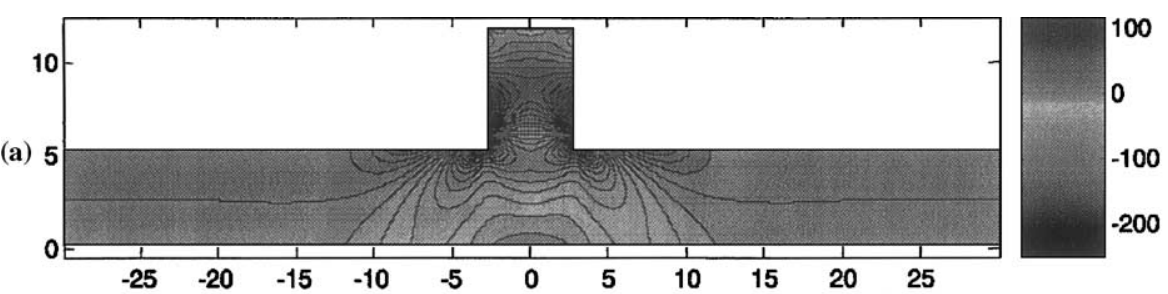

(b)

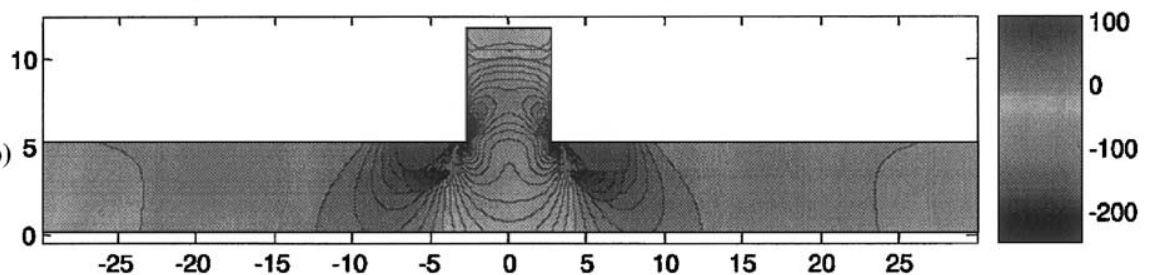

(c)
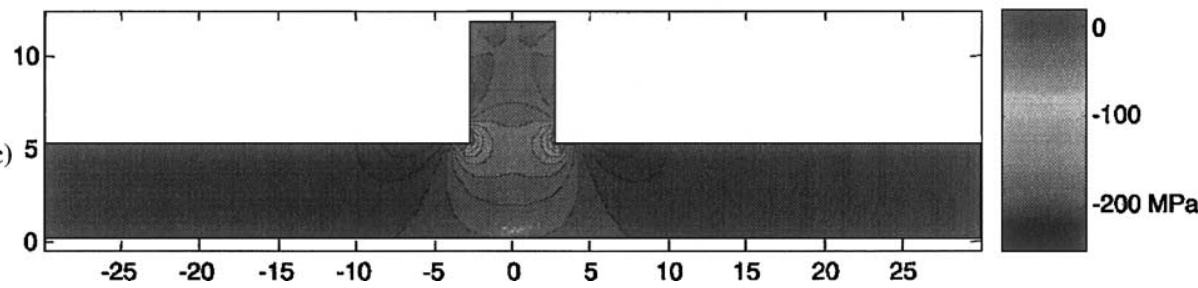

Fig. 1. Finite element results of the stress distributions in a ridge silicon waveguide core: (a) $\sigma_{x x}$; (b) $\sigma_{y y}$; (c) $\sigma_{z z}$. The stresses are induced by thermal mismatch of the top cladding layers. The unit of the stresses is MPa.

anisotropic. Fig. 2 shows the refractive index change caused by these stresses due to the photo-elastic effect. We can see that the stresses cause the inhomogeneous and anisotropic distribution of refractive indexes.

Stress birefringence has long been observed (Born and Wolf, 1964). Many efforts have been made to improve the optical performance by taking advantage of photo-elastic effect, such as stress release grooves to

(a)
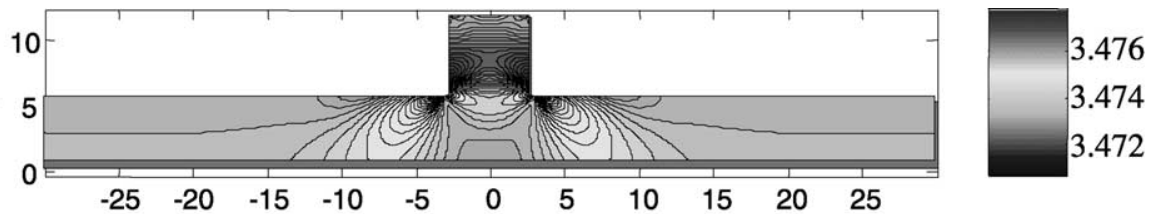

(b)
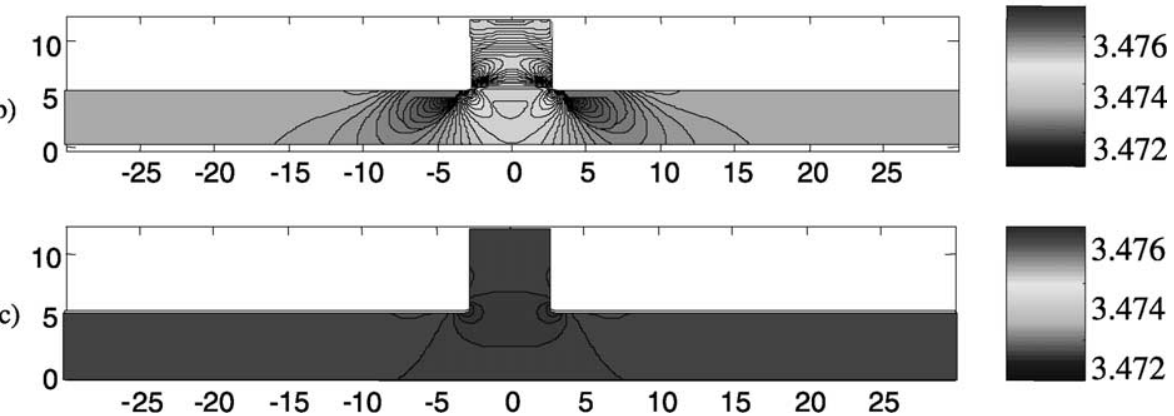

Fig. 2. Refractive index distributions caused by the stresses in Fig. 1: (a) $n_{x x}$; (b) $n_{y y}$; (c) $n_{z z}$. 
minimize the polarization shift (Nadler et al., 1999), thermal stress to control the temperature sensitivity of central wavelength (Cohen et al., 1996, 2000; Ooba et al., 2000; Huang and Yan, 2002), stress-induced optical waveguides and filters (Saitoh et al., 1999; Lea and Weiss, 1996; Almashary and Kim, 1996; Rho and Jackson, 1999; Savin et al., 2000), stress effects on laser (Maciejko et al., 1989, 1993), and stress-induced birefringence behavior of the waveguides and fibers (Kilian et al., 2000; Okuno et al., 1994; Chowdhury and Wilcox, 2000; Buda et al., 2000). However, stresses could also cause problems, such as the increments of polarization dependent loss (PDL) and polarization shift (PS) (Min et al., 2000; Yan et al., 2002). Although many observations have been made on the stress effects on the optical performance, systematical studies are rarely found.

In this paper, I illustrate the stress effects on the optical performance by using planar waveguides. The plan of this paper is as follows. Section 2 reviews the photo-elastic relation and wave equations of anisotropic and inhomogeneous waveguides. In Section 3, the effect index and mode shape for a planar waveguide are solved analytically under four stress states: hydrostatic, in-plane, stress concentration, and pure shear. Several practical concerns and the implications of stress effects are discussed in Section 4.

\section{Wave equations of anisotropic and inhomogeneous waveguides}

Considering fields in a region which contains no sources, the Maxwell's equations are

$$
\begin{aligned}
& \nabla \times \widetilde{E}=-\mu \frac{\partial \widetilde{H}}{\partial t}, \\
& \nabla \times \widetilde{H}=\varepsilon \frac{\partial \widetilde{E}}{\partial t},
\end{aligned}
$$

where $\nabla$ is the del operator, $\widetilde{E}$ is the electric field, $\widetilde{H}$ is the magnetic field, $\mu$ is the permeability and $\varepsilon$ is the permittivity of the medium and $t$ is time.

Considering a simple-harmonic time varying field, which can be expressed in the exponential form

$$
\begin{aligned}
& \widetilde{E}(x, y, z, t)=E(x, y, z) \exp (\mathrm{j} \omega t), \\
& \widetilde{H}(x, y, z, t)=H(x, y, z) \exp (\mathrm{j} \omega t),
\end{aligned}
$$

where $\omega$ is the optical frequency and $j=\sqrt{-1}$, the Maxwell's equations are transferred to

$$
\begin{aligned}
& \nabla \times E=-\mathrm{j} \omega \mu H, \\
& \nabla \times H=\mathrm{j} \omega \varepsilon E .
\end{aligned}
$$

The dielectric tensor takes the form of

$$
\varepsilon=\left(\begin{array}{ccc}
n_{x x}^{2} & n_{x y}^{2} & n_{x z}^{2} \\
n_{x y}^{2} & n_{y y}^{2} & n_{y z}^{2} \\
n_{x z}^{2} & n_{y z}^{2} & n_{z z}^{2}
\end{array}\right),
$$

where $n_{x x}, n_{y y}, n_{z z}, n_{x y}, n_{x z}$ and $n_{y z}$ are the refractive indexes, which are functions of $x, y$ and $z$. Due to the photo-elastic effect, the refractive indexes change with stress/strain. For media with cubic structure, the relation between the refractive index, $n$, and strain, $\gamma$, is expressed as (Xu and Stroud, 1992; Sapriel, 1979) 


$$
\Delta\left(\begin{array}{l}
1 / n_{x x}^{2} \\
1 / n_{y y}^{2} \\
1 / n_{z z}^{2} \\
1 / n_{y z}^{2} \\
1 / n_{x z}^{2} \\
1 / n_{x y}^{2}
\end{array}\right)=\left(\begin{array}{cccccc}
p_{11} & p_{12} & p_{12} & 0 & 0 & 0 \\
p_{12} & p_{11} & p_{12} & 0 & 0 & 0 \\
p_{12} & p_{12} & p_{11} & 0 & 0 & 0 \\
0 & 0 & 0 & p_{44} & 0 & 0 \\
0 & 0 & 0 & 0 & p_{44} & 0 \\
0 & 0 & 0 & 0 & 0 & p_{44}
\end{array}\right)\left(\begin{array}{c}
\gamma_{x x} \\
\gamma_{y y} \\
\gamma_{z z} \\
\gamma_{y z} \\
\gamma_{x z} \\
\gamma_{x y}
\end{array}\right)
$$

where $p_{11}, p_{12}$ and $p_{44}$ are strain-optic constants. By using the stress-strain relation, the relation between the index and the stress is found to be

$$
\left(\begin{array}{c}
n_{x x} \\
n_{y y} \\
n_{z z} \\
n_{y z} \\
n_{x z} \\
n_{x y}
\end{array}\right)=\left(\begin{array}{c}
n_{0} \\
n_{0} \\
n_{0} \\
0 \\
0 \\
0
\end{array}\right)-\left(\begin{array}{cccccc}
C_{1} & C_{2} & C_{2} & 0 & 0 & 0 \\
C_{2} & C_{1} & C_{2} & 0 & 0 & 0 \\
C_{2} & C_{2} & C_{1} & 0 & 0 & 0 \\
0 & 0 & 0 & C_{3} & 0 & 0 \\
0 & 0 & 0 & 0 & C_{3} & 0 \\
0 & 0 & 0 & 0 & 0 & C_{3}
\end{array}\right)\left(\begin{array}{c}
\sigma_{x x} \\
\sigma_{y y} \\
\sigma_{z z} \\
\sigma_{y z} \\
\sigma_{x z} \\
\sigma_{x y}
\end{array}\right)
$$

where $C_{1}=n_{0}^{3}\left(p_{11}-2 v p_{12}\right) /(2 E), C_{2}=n_{0}^{3}\left[p_{12}-v\left(p_{11}+p_{12}\right)\right] /(2 E)$ and $C_{3}=n_{0}^{3} p_{44} /(2 G)$ are stress-optic constants. $E, G$ and $v$ are Young's modulus, shear modulus and Poisson's ratio, respectively. For isotropic crystals, $p_{44}=\left(p_{11}-p_{12}\right) / 2$ and $G=E / 2 /(1+v)$. In Eq. (9), it is assumed that the refractive index of media is homogeneous and is in the system of principal dielectric axes at stress free state. Table 1 lists the photoelastic constants of some materials. Stress-optic constants, $C$, are calculated from strain-optic constants, $p$, listed in the book by $\mathrm{Xu}$ and Stroud (1992).

The stresses in microstructures are usually on the order of $10^{8} \mathrm{~Pa}$, and the $C$ values in Table 1 are on the order of $10^{-11} \mathrm{~Pa}^{-1}$; so the refractive index change caused by stress is normally between \pm 0.01 . Because the stresses are usually non-uniformly distributed (inhomogeneous) and have different values in different directions (anisotropic) in the waveguides, the refractive indexes are also inhomogeneous and anisotropic as shown in Figs. 1 and 2. As the waveguide is usually very long in one direction, denoted as $z$, the shear stresses in this direction can be ignored. Therefore, the dielectric tensor becomes

Table 1

\begin{tabular}{|c|c|c|c|c|c|c|c|c|}
\hline Material & $\lambda_{0}(\mu \mathrm{m})$ & $n_{0}$ & $p_{11}$ & $p_{12}$ & $p_{44}$ & $C_{1}\left(10^{-12} / \mathrm{Pa}\right)$ & $C_{2}\left(10^{-12} / \mathrm{Pa}\right)$ & $C_{3}\left(10^{-12} / \mathrm{Pa}\right)$ \\
\hline \multirow[t]{2}{*}{$\mathrm{Ge}$} & $2.0-2.2$ & 4 & -0.063 & -0.0535 & -0.074 & -10.56 & -6.78 & -35.29 \\
\hline & 10.6 & & 0.27 & 0.235 & 0.125 & 44.27 & 30.37 & 59.61 \\
\hline \multirow[t]{2}{*}{$\mathrm{Si}$} & 1.15 & 3.42 & -0.101 & 0.0094 & & -11.35 & 3.65 & \\
\hline & 3.39 & & -0.094 & 0.017 & -0.051 & -11.04 & 4.04 & -12.82 \\
\hline GaAs & 1.15 & 3.43 & -0.165 & -0.140 & -0.072 & -18.39 & -10.63 & -24.46 \\
\hline $\begin{array}{l}\text { Fused } \\
\text { silica }\end{array}$ & 0.633 & 1.46 & 0.121 & 0.270 & & 0.65 & 4.50 & -3.85 \\
\hline $\mathrm{GaP}$ & 0.633 & 3.32 & -0.151 & -0.082 & -0.074 & -17.91 & -1.87 & -19.21 \\
\hline $\mathrm{LiNbO}_{3}$ & 0.633 & 2.29 & -0.026 & 0.090 & 0.146 & -2.10 & 2.55 & -14.63 \\
\hline $\mathrm{LiTaO} 3$ & 0.633 & 2.18 & -0.084 & 0.081 & 0.028 & -2.57 & 1.91 & 1.53 \\
\hline $\mathrm{Al}_{2} \mathrm{O}_{3}$ & 0.633 & 1.76 & -0.23 & -0.03 & -0.10 & -1.61 & 0.202 & -1.90 \\
\hline $\mathrm{PbMoO}_{4}$ & 0.633 & 2.39 & 0.24 & 0.24 & 0.067 & 6.63 & 6.63 & 17.04 \\
\hline
\end{tabular}

The photo-elastic constants of some materials 


$$
\varepsilon=\left(\begin{array}{ccc}
n_{x x}^{2} & n_{x y}^{2} & 0 \\
n_{x y}^{2} & n_{y y}^{2} & 0 \\
0 & 0 & n_{z z}^{2}
\end{array}\right) .
$$

In order to solve Eqs. (5) and (6), we only need to consider two special cases: (1) $H_{z}=0$ and (2) $E_{z}=0$. Any field distribution can be expressed as a combination of the field distributions of the different modes obtained in the two special cases. For the first case, the magnetic field is transverse to the direction of propagation; such modes are known as transverse magnetic (or TM) modes. For the second case, the electric field is transverse to the direction of propagation; such modes are known as transverse electric (or TE) modes.

Applying the following transformation:

$$
\begin{aligned}
& E(x, y, z)=e(x, y, z) \exp \left(-\mathrm{j} n_{e} k z\right), \\
& H(x, y, z)=h(x, y, z) \exp \left(-\mathrm{j} n_{h} k z\right),
\end{aligned}
$$

gives the wave equations in terms of TE fields for anisotropic and inhomogeneous waveguides (Xu et al., 1994)

$$
\left\{\begin{array}{ll}
P_{x x} & P_{x y} \\
P_{y x} & P_{y y}
\end{array}\right\}\left\{\begin{array}{l}
e_{x} \\
e_{y}
\end{array}\right\}=\mathrm{j} 2 n_{e} k \frac{\partial}{\partial z}\left\{\begin{array}{l}
e_{x} \\
e_{y}
\end{array}\right\}
$$

where $k=2 \pi / \lambda, n_{e}$ is the effective refractive index of TE mode, and

$$
\begin{aligned}
& P_{x x} e_{x}=\frac{\partial}{\partial x}\left[\frac{1}{n_{z z}^{2}} \frac{\partial}{\partial x}\left(n_{x x}^{2} e_{x}\right)\right]+\frac{\partial^{2} e_{y}}{\partial y^{2}}+k^{2}\left(n_{x x}^{2}-n_{e}^{2}\right) e_{x}, \\
& P_{y y} e_{y}=\frac{\partial}{\partial y}\left[\frac{1}{n_{z z}^{2}} \frac{\partial}{\partial y}\left(n_{y y}^{2} e_{y}\right)\right]+\frac{\partial^{2} e_{y}}{\partial x^{2}}+k^{2}\left(n_{y y}^{2}-n_{e}^{2}\right) e_{y}, \\
& P_{x y} e_{y}=n_{x y}^{2} k^{2} e_{y}+\frac{\partial}{\partial x}\left[\frac{1}{n_{z z}^{2}} \frac{\partial}{\partial y}\left(n_{y y}^{2} e_{y}\right)\right]-\frac{\partial^{2} e_{y}}{\partial x \partial y}, \\
& P_{y x} e_{x}=n_{x y}^{2} k^{2} e_{x}+\frac{\partial}{\partial y}\left[\frac{1}{n_{z z}^{2}} \frac{\partial}{\partial x}\left(n_{x x}^{2} e_{x}\right)\right]-\frac{\partial^{2} e_{x}}{\partial y \partial x} .
\end{aligned}
$$

Similarly, we can derive the wave equations in terms of TM fields for anisotropic and inhomogeneous waveguides as

$$
\left\{\begin{array}{ll}
Q_{x x} & Q_{x y} \\
Q_{y x} & Q_{y y}
\end{array}\right\}\left\{\begin{array}{l}
h_{x} \\
h_{y}
\end{array}\right\}=\mathrm{j} 2 n_{h} k \frac{\partial}{\partial z}\left\{\begin{array}{l}
h_{x} \\
h_{y}
\end{array}\right\}
$$

where $n_{h}$ is the effective refractive index of TM mode, and

$$
\begin{aligned}
Q_{x x} h_{x} & =\frac{\partial^{2} h_{x}}{\partial x^{2}}+n_{y y}^{2} \frac{\partial}{\partial y}\left(\frac{1}{n_{z z}^{2}} \frac{\partial h_{x}}{\partial y}\right)+k^{2}\left(n_{y y}^{2}-n_{e}^{2}\right) h_{x}, \\
Q_{y y} h_{y} & =\frac{\partial^{2} h_{y}}{\partial y^{2}}+n_{x x}^{2} \frac{\partial}{\partial x}\left(\frac{1}{n_{z z}^{2}} \frac{\partial h_{y}}{\partial x}\right)+k^{2}\left(n_{x x}^{2}-n_{e}^{2}\right) h_{y} \\
Q_{x y} h_{y} & =n_{x y}^{2} k^{2} h_{y}+\frac{\partial^{2} h_{y}}{\partial y \partial x}-n_{y y}^{2} \frac{\partial}{\partial y}\left(\frac{1}{n_{z z}^{2}} \frac{\partial h_{y}}{\partial x}\right) \\
Q_{y x} h_{x} & =n_{x y}^{2} k^{2} h_{x}+\frac{\partial^{2} h_{x}}{\partial x \partial y}-n_{x x}^{2} \frac{\partial}{\partial x}\left(\frac{1}{n_{z z}^{2}} \frac{\partial h_{x}}{\partial y}\right)
\end{aligned}
$$


To obtain the TE and TM modes, the field is considered to be independent of $z$ direction. The mode equations are

$$
\begin{aligned}
& \left\{\begin{array}{ll}
P_{x x} & P_{x y} \\
P_{y x} & P_{y y}
\end{array}\right\}\left\{\begin{array}{l}
e_{x} \\
e_{y}
\end{array}\right\}=0, \\
& \left\{\begin{array}{ll}
Q_{x x} & Q_{x y} \\
Q_{y x} & Q_{y y}
\end{array}\right\}\left\{\begin{array}{l}
h_{x} \\
h_{y}
\end{array}\right\}=0 .
\end{aligned}
$$

These are eigenvalue equations. By using the boundary conditions, we can obtain the eigenvalues, $n_{e}$ and $n_{h}$, and eigenvectors, $e$ and $h$. This eigenvalue problem can only be solved numerically for most practical waveguide structures. In order to illustrate the stress effects, in the next section, we will study a simple structure, a planar waveguide, and analytically solve this problem.

\section{A planar waveguide}

A planar waveguide consists of an infinitely large planar core with the thickness on the order of the wavelength and a higher refractive index than the surrounding cladding regions. Fig. 3 shows the schematic of a symmetric three-layer planar optic waveguide. For the planar waveguides, the light propagates in the $z$ direction, is confined in the $x$ direction within the central core region, and has no variation in the $y$ direction. Although most practical optical waveguides are channel waveguides, where the light is also confined in the $y$ direction, planar waveguides are used to provide a basic understanding since analytical solutions may be obtained for this kind of structure.

In the planar waveguides, the fields are independent of the $y$ coordinate. By using Eq. (6) and $E_{z}=0$, the TE mode equations (16) can be simplified to

$$
\mathrm{TE}: \frac{\mathrm{d}^{2} e_{y}}{\mathrm{~d} x^{2}}+k^{2}\left(n_{y y}^{2}-n_{e}^{2}-\frac{n_{x y}^{4}}{n_{x x}^{2}-n_{e}^{2}}\right) e_{y}=0 .
$$

Similarly, by using Eq. (5) and $H_{z}=0$, the TM mode equations (17) can be simplified to

$$
\mathrm{TM}: \quad \frac{\mathrm{d}^{2} h_{y}}{\mathrm{~d} x^{2}}+p \frac{\mathrm{d} h_{y}}{\mathrm{~d} x}+k^{2} \frac{n_{z z}^{2}}{n_{x x}^{2}}\left(n_{x x}^{2}-n_{h}^{2}-\frac{n_{x y}^{4}}{n_{y y}^{2}-n_{e}^{2}}\right) h_{y}=0,
$$

where

$$
p(x)=\frac{-2}{n_{z z}} \frac{\mathrm{d} n_{z z}}{\mathrm{~d} x} .
$$

Solving the above equations, we can obtain the TE and TM modes. To focus on the stress effects, in the follow discussions, we will study a simple structure: a symmetric three-layer planar optical waveguide with

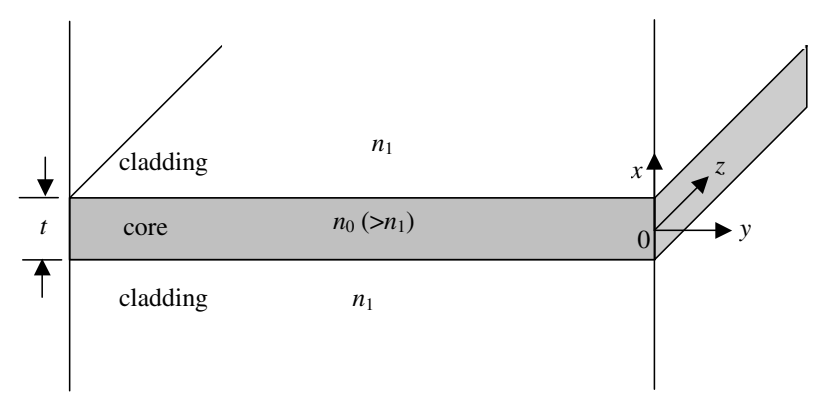

Fig. 3. Schematic of a symmetric three-layer planer waveguide. 
infinitely thick cladding regions (Fig. 3), and apply four kinds of stress states: hydrostatic, in-plane, stress concentration, and pure shear. To simplify the problem and emphasize the stress effects on the core, we ignore the photo-elastic effects of the cladding region. The refractive index of the cladding region, $n_{1}$, is assumed to be a constant when applying loads. In this paper, we take $n_{0}=3.5, n_{1}=3.4, \lambda=0.83 \mu \mathrm{m}$ and $C_{2} / C_{1}=0.1$ in all the analyses. For other planar waveguides with more complicated structure subjected to more complicated stresses, similar method can be used.

\subsection{Hydrostatic stress state}

In order to study the stress magnitude effect, the core is assumed to be under hydrostatic stress state, i.e., $\sigma_{x x}=\sigma_{y y}=\sigma_{z z}=\sigma$ and $\sigma_{x y}=0$. Therefore, the index of the core is isotropic and homogeneous, and the value of the refractive index in the core changes due to the stress, i.e., $n_{x x}=n_{y y}=n_{z z}=n=n_{0}-$ $\left(C_{1}+2 C_{2}\right) \sigma$. The mode equations of the core are simplified to

$\mathrm{TE}: \quad \frac{\mathrm{d}^{2} e_{y}}{\mathrm{~d} x^{2}}+k^{2}\left(n^{2}-n_{e}^{2}\right) e_{y}=0$

$\mathrm{TM}: \quad \frac{\mathrm{d}^{2} h_{y}}{\mathrm{~d} x^{2}}+k^{2}\left(n^{2}-n_{h}^{2}\right) h_{y}=0$.

By using the boundary condition of zero fields at infinite large $x$, the above equations can be solved, and the solutions are listed in Table 2.

At the interface between core and cladding, the boundary conditions are (Boyd, 1994):

TE: $e_{y}$ and $h_{z} \sim \frac{\mathrm{d} e_{y}}{\mathrm{~d} x}$ continuous across the boundary.

TM: $h_{y}$ and $e_{z} \sim\left(\frac{1}{n^{2}}\right) \frac{\mathrm{d} h_{y}}{\mathrm{~d} x}$ continuous across the boundary

These are eigenvalue problems. $A, B, C$ and $D$ are constants. Applying the above boundary conditions results in four homogeneous linear equations involving the four constants for each mode. For these linear equations to have nontrivial solutions for the constants, the determinant of the coefficients must equal zero. From this relation, the effective indexes, $n_{e}$ and $n_{h}$, can be determined. Fig. 4 shows the TM effective refractive index, $n_{h}$, as a function of core thickness. Fig. 5 shows the effective indexes as a function of normalized stress for a $1 \mu \mathrm{m}$ core thickness waveguide. The TM effective index is very close to the TE effective index under this stress state. However, the effective indexes vary with stress, and the cutoff thickness, which is defined as the core thickness at which the field is no longer guided by the core ( $n_{e}=n_{h}=n_{1}$ in this case), shifts when the stress value is high. In Fig. 5, when $t=1 \mu \mathrm{m}$, the second mode $(m=2)$ appears or disappears when stress changes. This means that stress can cause multimode. The field distributions of the fundamental modes $(m=0)$ are shown in Fig. 6 . The modal field is normalized by the time-averaged power flow, $P$, which is given by the integral over the waveguide cross-section of the $z$-component of the Poynting vector, $\frac{1}{2} \operatorname{Re}\left(E \times H^{*}\right)_{z}$ (Adams, 1981):

Table 2

Solutions for TE and TM modes under hydrostatic stress state

\begin{tabular}{lll}
\hline Region & TE solutions & TM solutions \\
\hline$x \geqslant t / 2$ & $A_{E} \exp \left(-k x \sqrt{n_{e}^{2}-n_{1}^{2}}\right)$ & $A_{M} \exp \left(-k x \sqrt{n_{h}^{2}-n_{1}^{2}}\right)$ \\
$t / 2>|x|$ & $B_{E} \cos \left(k x \sqrt{n^{2}-n_{e}^{2}}\right)+C_{E} \sin \left(k x \sqrt{n^{2}-n_{e}^{2}}\right)$ & $B_{M} \cos \left(k x \sqrt{n^{2}-n_{h}^{2}}\right)+C_{M} \sin \left(k x \sqrt{n^{2}-n_{h}^{2}}\right)$ \\
$-t / 2 \geqslant x$ & $D_{E} \exp \left(k x \sqrt{n_{e}^{2}-n_{1}^{2}}\right)$ & $D_{M} \exp \left(k x \sqrt{n_{h}^{2}-n_{1}^{2}}\right)$ \\
\hline
\end{tabular}




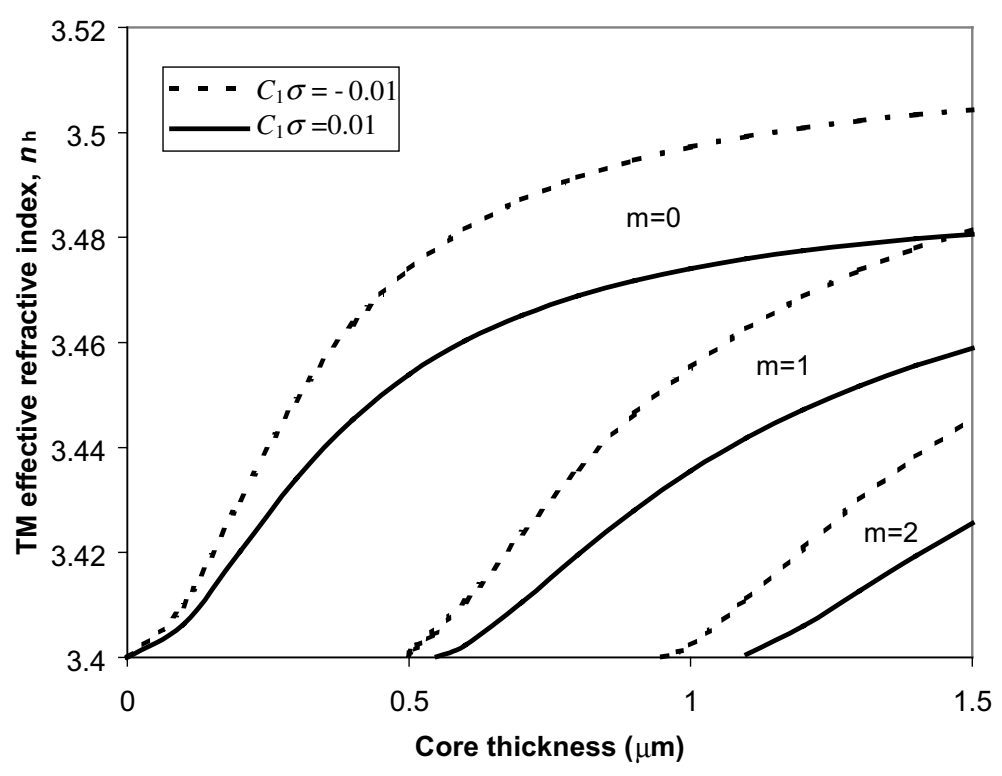

Fig. 4. TM effective refractive index, $n_{h}$, as a function of core thickness for a symmetric planar optical waveguide under different hydrostatic stresses.

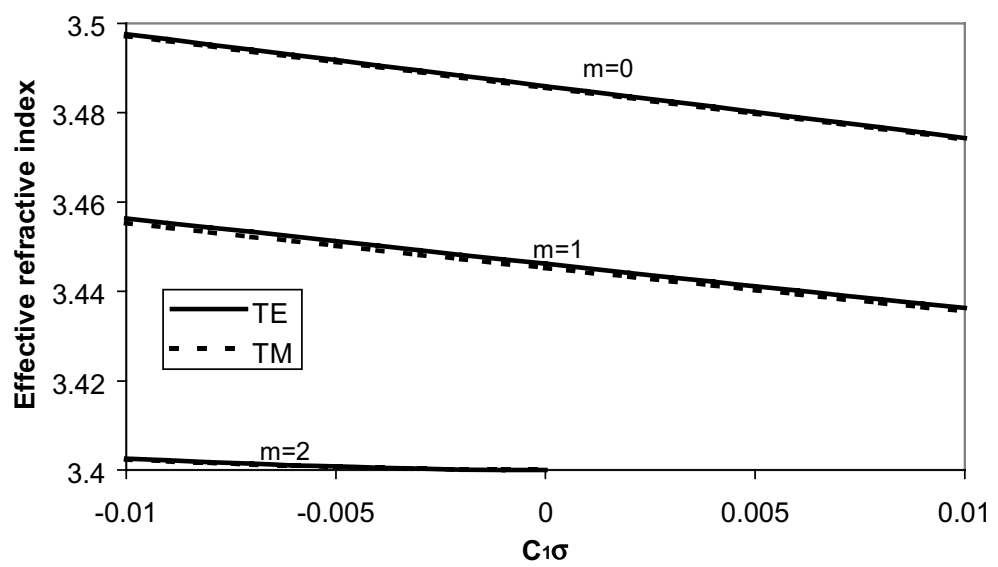

Fig. 5. Effective refractive index as a function of normalized hydrostatic stress for a symmetric planar optical waveguide with $t=1 \mu \mathrm{m}$.

$$
P=\frac{1}{2} \int_{-\infty}^{\infty} \operatorname{Re}\left(E \times H^{*}\right)_{z} \mathrm{~d} x
$$

The mode shape changes little under the hydrostatic stress.

\subsection{In-plane stress state}

To study the stress anisotropy effect, the core is considered under in-plan stress state, i.e., $\sigma_{x x}=0, \sigma_{x y}=0$ and $\sigma_{y y}=\sigma_{z z}=\sigma$. From Eq. (9), the refractive index in the core is 


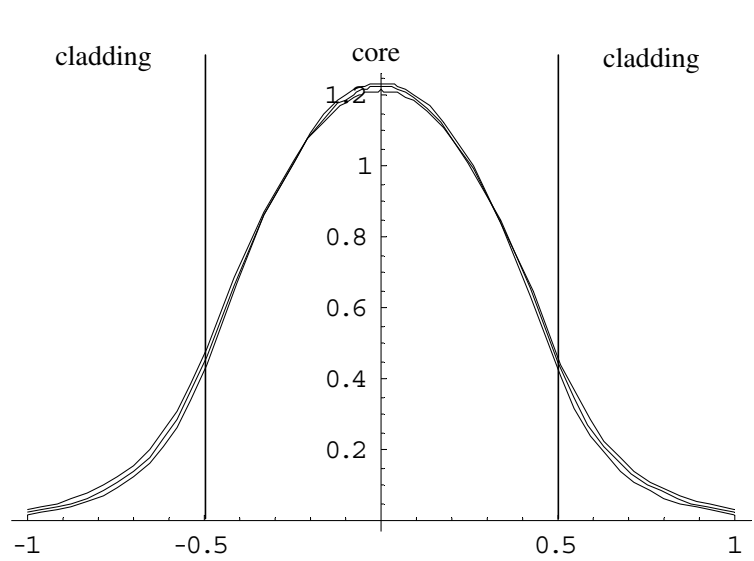

(a)

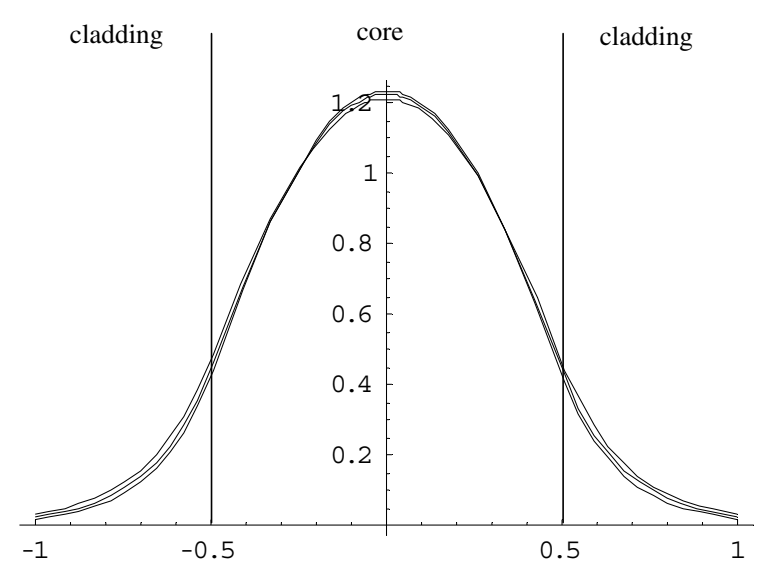

(b)

Fig. 6. Normalized transverse field distribution of the fundamental mode $(m=0)$ for a symmetric optical waveguide under different hydrostatic stresses with $t=1 \mu \mathrm{m}$ : (a) TM mode; (b) TE mode. The curves from top are corresponding to $C_{1} \sigma=-0.01, C_{1} \sigma=0$ and $C_{1} \sigma=0.01$ stress values.

$$
\left(\begin{array}{c}
n_{x x} \\
n_{y y} \\
n_{z z}
\end{array}\right)=\left(\begin{array}{c}
n_{0}-2 C_{2} \sigma \\
n_{0}-\left(C_{1}+C_{2}\right) \sigma \\
n_{0}-\left(C_{1}+C_{2}\right) \sigma
\end{array}\right) .
$$

The mode equations of the core are simplified to

$$
\begin{aligned}
& \text { TE: } \quad \frac{\mathrm{d}^{2} e_{y}}{\mathrm{~d} x^{2}}+k^{2}\left(n_{y y}^{2}-n_{e}^{2}\right) e_{y}=0 \\
& \mathrm{TM}: \quad \frac{\mathrm{d}^{2} h_{y}}{\mathrm{~d} x^{2}}+k^{2}\left(n_{z z}^{2}-n_{z z}^{2} n_{h}^{2} / n_{x x}^{2}\right) h_{y}=0 .
\end{aligned}
$$

By using the boundary condition that at infinitely large $x$, the material carries no fields, the above equations can be solved, and the solutions are listed in Table 3.

At the interface between core and cladding, the boundary conditions are:

TE: $e_{y}$ and $\frac{\mathrm{d} e_{y}}{\mathrm{~d} x}$ continuous across the boundary

TM: $h_{y}$ and $\left(\frac{1}{n_{z z}^{2}}\right) \frac{\mathrm{d} h_{y}}{\mathrm{~d} x}$ continuous across the boundary

Table 3

Solutions for TE and TM modes under in-plane stress state

\begin{tabular}{lll}
\hline Region & TE solutions & TM solutions \\
\hline$x \geqslant t / 2$ & $A_{E} \exp \left(-k x \sqrt{n_{e}^{2}-n_{1}^{2}}\right)$ & $A_{M} \exp \left(-k x \sqrt{n_{z z}^{2} n_{h}^{2} / n_{x x}^{2}-n_{1}^{2}}\right)$ \\
$t / 2>|x|$ & $B_{E} \cos \left(k x \sqrt{n_{y y}^{2}-n_{e}^{2}}\right)+C_{E} \sin \left(k x \sqrt{n_{y y}^{2}-n_{e}^{2}}\right)$ & $B_{M} \cos \left(k x \sqrt{n_{z z}^{2}-n_{z z}^{2} h_{h}^{2} / n_{x x}^{2}}\right)+C_{M} \sin \left(k x \sqrt{n_{z z}^{2}-n_{z z}^{2} h_{h}^{2} / n_{x x}^{2}}\right)$ \\
$-t / 2 \geqslant x$ & $D_{E} \exp \left(k x \sqrt{n_{e}^{2}-n_{1}^{2}}\right)$ & $D_{M} \exp \left(k x \sqrt{n_{z z}^{2} n_{h}^{2} / n_{x x}^{2}-n_{1}^{2}}\right)$ \\
\hline
\end{tabular}


Solving these eigenvalue problems, we can obtain the effective refractive index and normalized mode shape. Fig. 7 shows the effective index as a function of core thickness. Fig. 8 shows the effective indexes as a function of normalized stress. The normalized field distributions of the fundamental mode $(m=0)$ are shown in Fig. 9. The plots of in-plane stress state are similar to those of hydrostatic stress state, except that the refractive indexes of TM mode are quite different from those of TE mode due to stress anisotropy. The in-plane stress can cause birefringence, which will be discussed later.

\subsection{Stress concentration}

To study the inhomogeneous stress effect, we consider a concentrated stress distribution. Stress is assumed to be isotropic and concentrated between $-\delta$ and $+\delta$ at the center of the core, and refractive indexes do not change with stresses in other regions. Assuming the stress field in the core as
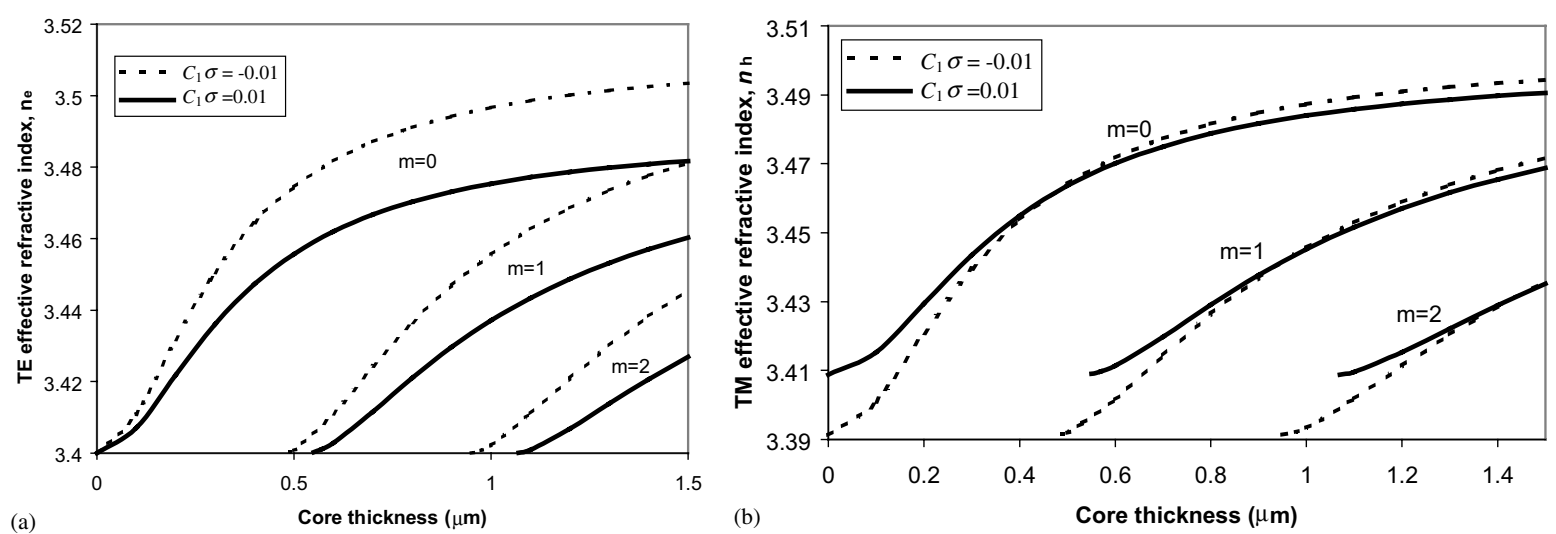

Fig. 7. Effective refractive index as a function of core thickness for a symmetric planar optical waveguide under different in-plane stresses: (a) TE mode; (b) TM mode.

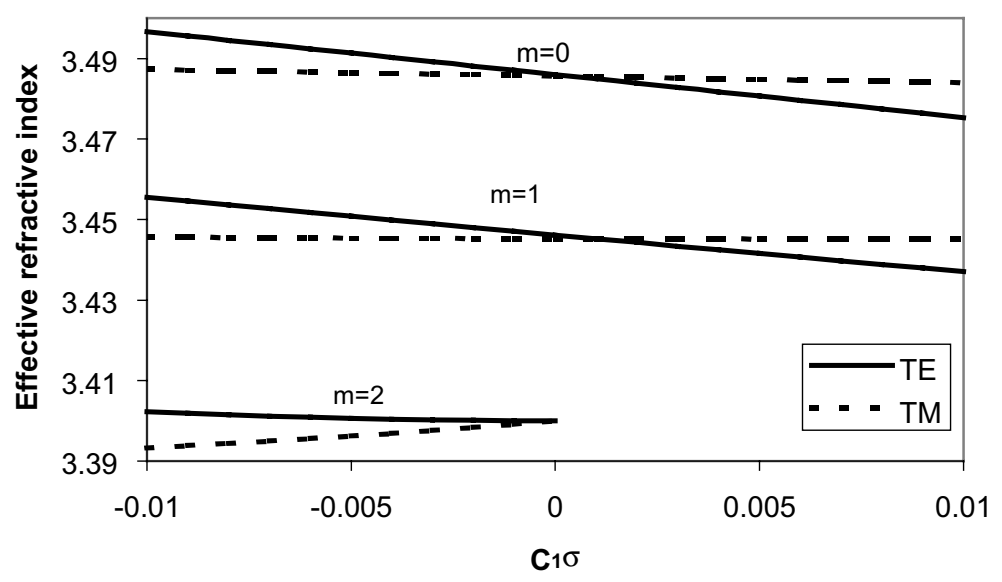

Fig. 8. Effective refractive index as a function of normalized in-plane stress for a symmetric planar optical waveguide with $t=1 \mu \mathrm{m}$. 


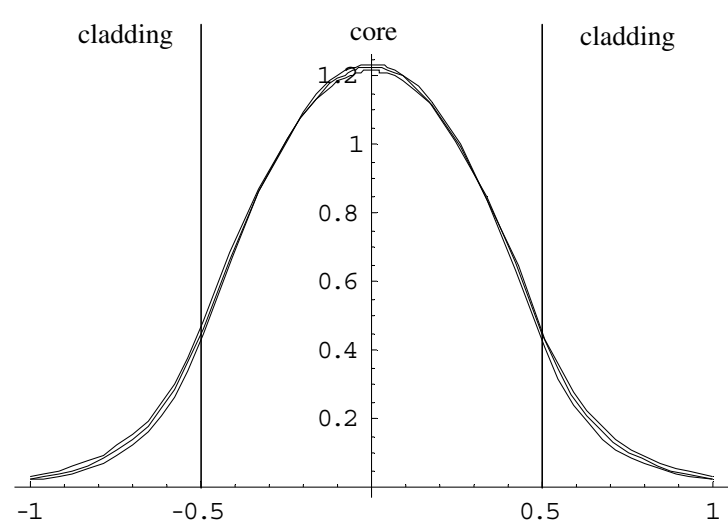

(a)

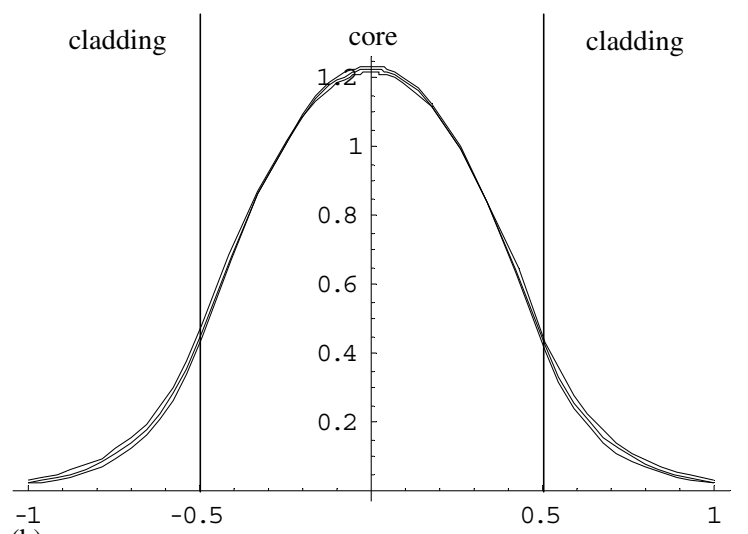

(b)

Fig. 9. Normalized transverse field distribution of the fundamental mode $(m=0)$ for a symmetric optical waveguide under different inplane stresses with $t=1 \mu \mathrm{m}$ : (a) TM mode; (b) TE mode. The curves from top are corresponding to $C_{1} \sigma=-0.01, C_{1} \sigma=0$ and $C_{1} \sigma=0.01$ stress values.

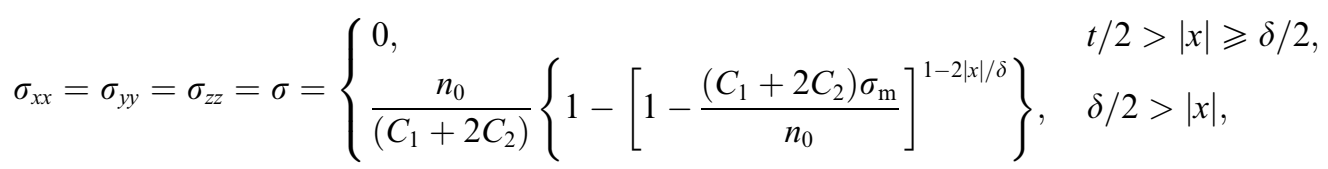

the corresponding refractive indexes in the core under this stress state are

$$
n_{x x}=n_{y y}=n_{z z}=n= \begin{cases}n_{0}, & t / 2>|x| \geqslant \delta / 2, \\ n_{0}\left[1-\frac{\left(C_{1}+2 C_{2}\right) \sigma_{\mathrm{m}}}{n_{0}}\right]^{1-2|x| / \delta}, & \delta / 2>|x|\end{cases}
$$

where $\sigma_{\mathrm{m}}$ is the maximum stress at the middle of the core, which is a constant. By using this index profile (27), the mode equations in the stress concentration area are

TE: $\quad \frac{\mathrm{d}^{2} e_{y}}{\mathrm{~d} x^{2}}+k^{2}\left(n^{2}-n_{e}^{2}\right) e_{y}=0$,

$\mathrm{TM}: \quad \frac{\mathrm{d}^{2} h_{y}}{\mathrm{~d} x^{2}}+\operatorname{sign}(x) p_{\mathrm{m}} \frac{\mathrm{d} h_{y}}{\mathrm{~d} x}+k^{2}\left(n^{2}-n_{h}^{2}\right) h_{y}=0$,

where $p_{\mathrm{m}}=4 \ln \left[1-\sigma_{\mathrm{m}}\left(C_{1}+2 C_{2}\right) / n_{0}\right] / \delta$. Denoting $\phi=e_{y}, \quad \gamma=n_{e} \quad$ for $\quad \mathrm{TE} ; \quad$ and $\phi=h_{y} / n, \quad \gamma=$ $\sqrt{n_{h}^{2}+p_{\mathrm{m}}^{2} /\left(4 k^{2}\right)}$ for TM, the mode equations for TE (28) and TM (29) can be combined together as (Owyang, 1981; Sodha and Ghatak, 1977)

$$
\frac{\mathrm{d}^{2} \phi}{\mathrm{d} x^{2}}+k^{2}\left(n^{2}-\gamma^{2}\right) \phi=0
$$

Eq. (30) can be further transformed to

$$
\xi^{2} \frac{\mathrm{d}^{2} \phi}{\mathrm{d} \xi^{2}}+\xi \frac{\mathrm{d} \phi}{\mathrm{d} \xi}+\left(\xi^{2}-\alpha^{2}\right) \phi=0
$$

where $\xi=2 \mathrm{kn} / p_{\mathrm{m}}$ and $\alpha=2 \mathrm{k \gamma} / \mathrm{p}_{\mathrm{m}}$. This is a Bessel's equation. The solution is a linear combination of $J_{\alpha}(\xi)$ and $Y_{\alpha}(\xi)$, where $J_{\alpha}$ and $Y_{\alpha}$ are the Bessel functions of the first kind and second kind, respectively. The solutions of the mode equations are listed in Table 4. 
Table 4

Solutions for TE and TM modes under stress concentration state

\begin{tabular}{lll}
\hline Region & TE solutions & TM solutions \\
\hline$x \geqslant t / 2$ & $A_{E} \exp \left(-k \sqrt{n_{e}^{2}-n_{1}^{2}} x\right)$ & $A_{M} \exp \left(-k \sqrt{n_{h}^{2}-n_{1}^{2}} x\right)$ \\
$t / 2>x \geqslant \delta / 2$ & $B_{E} \cos \left(k \sqrt{n_{0}^{2}-n_{e}^{2}} x\right)+C_{E} \sin \left(k \sqrt{n_{0}^{2}-n_{e}^{2}} x\right)$ & $B_{M} \cos \left(k \sqrt{n_{0}^{2}-n_{h}^{2}} x\right)+C_{M} \sin \left(k \sqrt{n_{0}^{2}-n_{h}^{2}} x\right)$ \\
$\delta / 2>|x|$ & $D_{E} J_{\alpha E}\left(2 k n / p_{\mathrm{m}}\right)+E_{E} Y_{\alpha E}\left(2 k n / p_{\mathrm{m}}\right)$ & $n\left[D_{M} J_{\alpha M}\left(2 k n / p_{\mathrm{m}}\right)+E_{M} Y_{\alpha M}\left(2 k n / p_{\mathrm{m}}\right)\right]$ \\
$-\delta / 2>x \geqslant-t / 2$ & $F_{E} \cos \left(k \sqrt{n_{0}^{2}-n_{e}^{2}} x\right)+G_{E} \sin \left(k \sqrt{n_{0}^{2}-n_{e}^{2}} x\right)$ & $F_{M} \cos \left(k \sqrt{n_{0}^{2}-n_{h}^{2}} x\right)+G_{M} \sin \left(k \sqrt{n_{0}^{2}-n_{h}^{2}} x\right)$ \\
$-t / 2 \geqslant x$ & $H_{E} \exp \left(k \sqrt{n_{e}^{2}-n_{1}^{2}} x\right)$ & $H_{M} \exp \left(k \sqrt{n_{h}^{2}-n_{1}^{2}} x\right)$ \\
\hline
\end{tabular}

Where $\alpha E=2 k n_{e} / p_{\mathrm{m}}$ and $\alpha M=\sqrt{4 k^{2} n_{h}^{2} / p_{\mathrm{m}}^{2}+1}$.

Applying interface boundary conditions similar as the hydrostatic stress state, and solving these eigenvalue equations, we can obtain the effective refractive index and normalized mode shape. Fig. 10 shows the TM effective refractive index, $n_{h}$, as a function of waveguide thickness. Fig. 11 shows the effective indexes as a function of normalized stress. The normalized field distributions of the fundamental mode $(m=0)$ are shown in Fig. 12. In these figures, we take $\delta / t=0.05$, and $\sigma_{\mathrm{m}} C_{1}$ is considered between \pm 0.1 as the maximum stress in the stress concentration area is very high. It is important to note that the mode shape changes a lot when stresses concentrate. The reason is that stress concentration causes the non-uniform distribution of the refractive index, and the light trends to propagate in the higher refractive index region. From Figs. 10 and 11, we can see that stresses have much less effect on the first mode $(m=1)$ than on other modes in this case. Because the waveguide considered here is symmetric, the field of the first mode is zero at the center of the core, where the stress concentration happens.

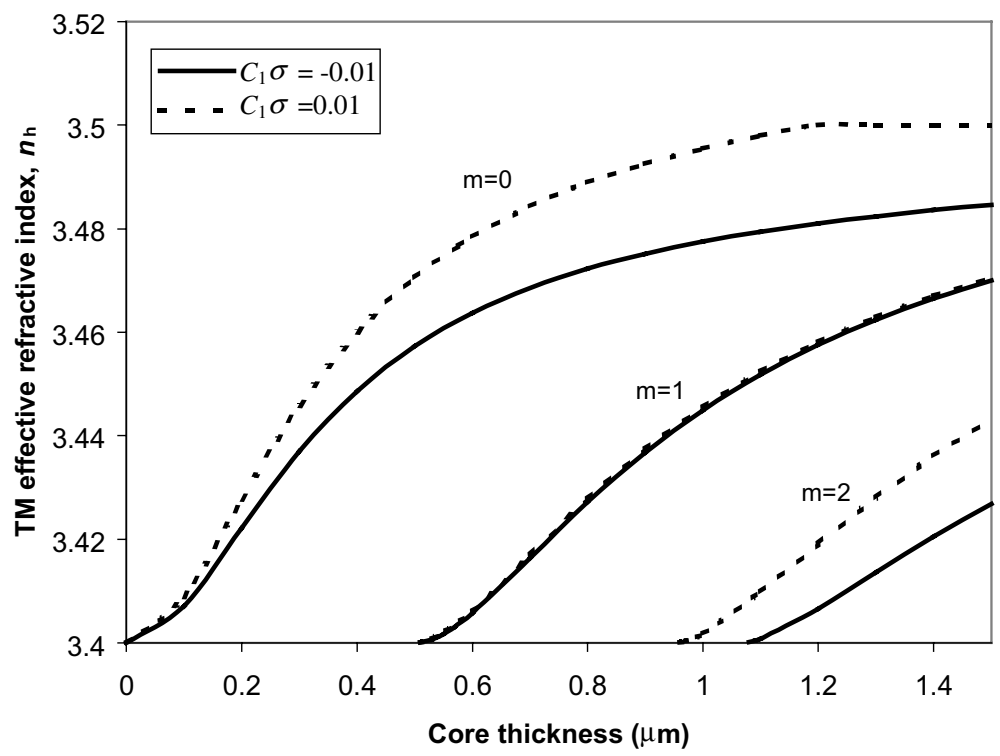

Fig. 10. TM effective refractive index, $n_{h}$, as a function of core thickness for a symmetric planar optical waveguide under different concentrated stresses. 


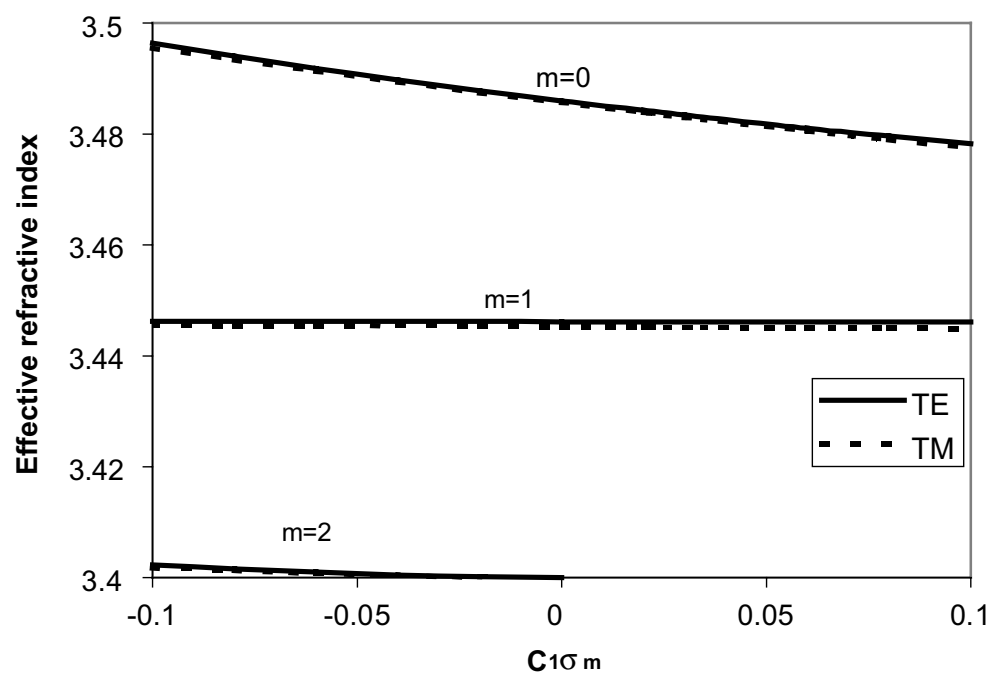

Fig. 11. Effective refractive index as a function of normalized concentrated stress for a symmetric planar optical waveguide with $t=$ $1 \mu \mathrm{m}$.

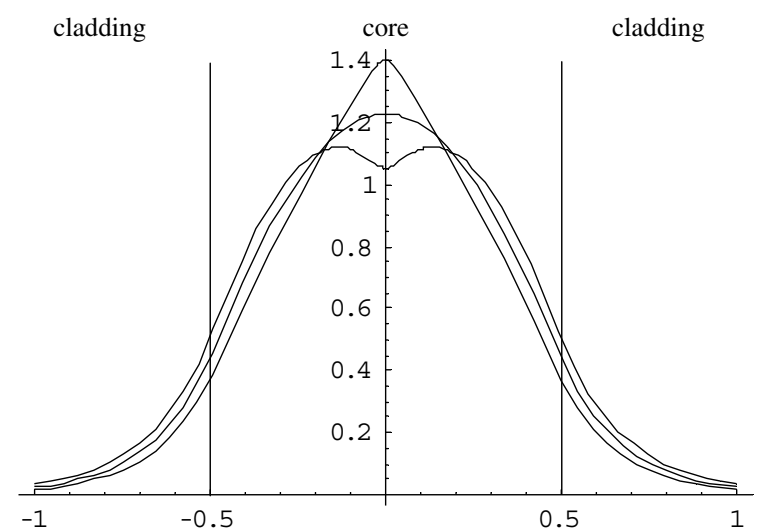

(a)

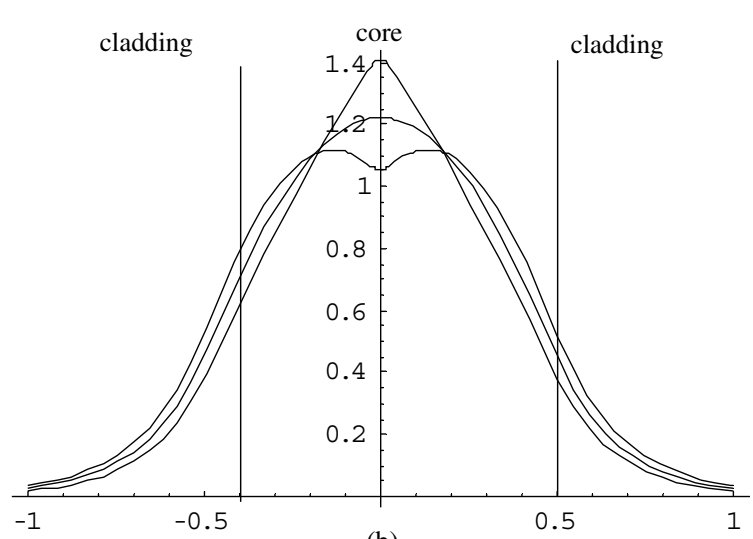

(b)

Fig. 12. Normalized transverse field distribution of the fundamental mode $(m=0)$ for a symmetric optical waveguide under different concentrated stresses with $t=1 \mu \mathrm{m}$ : (a) TM mode; (b) TE mode. The curves from top are corresponding to $C_{1} \sigma_{\mathrm{m}}=-0.1, C_{1} \sigma_{\mathrm{m}}=0$ and $C_{1} \sigma_{\mathrm{m}}=0.1$ stress values.

\subsection{Pure shear stress state}

To study the shear stress effect, the core is assumed to be under pure shear stress state, i.e., $\sigma_{x x}=$ $\sigma_{y y}=\sigma_{z z}=0$ and $\sigma_{x y}=\tau$. Therefore, the index of the core is isotropic and homogeneous, and the value of the index, $n_{x y}$, changes due to the shear stress, i.e., $n_{x y}=-C_{3} \tau$. The mode equations of the core are

$\mathrm{TE}: \quad \frac{\mathrm{d}^{2} e_{y}}{\mathrm{~d} x^{2}}+k^{2}\left[n_{0}^{2}-n_{e}^{2}-n_{x y}^{4} /\left(n_{0}^{2}-n_{e}^{2}\right)\right] e_{y}=0$, 


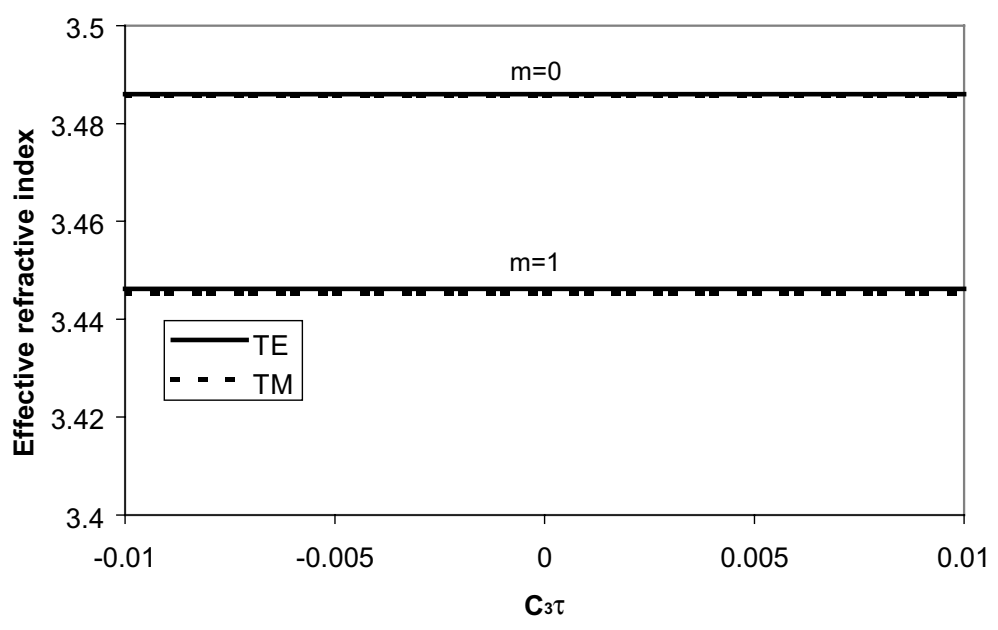

Fig. 13. Effective refractive index as a function of normalized pure shear stress for a symmetric planar optical waveguide with $t=1 \mu \mathrm{m}$.

$$
\mathrm{TM}: \quad \frac{\mathrm{d}^{2} h_{y}}{\mathrm{~d} x^{2}}+k^{2}\left[n_{0}^{2}-n_{h}^{2}-n_{x y}^{4} /\left(n_{0}^{2}-n_{h}^{2}\right)\right] h_{y}=0 .
$$

Using the similar method and boundary conditions as the hydrostatic stress state, we solved the eigenvalue problems and obtained the effective refractive index (Fig. 13). Pure shear stress has very little effect on the optical performance.

\section{Several practical issues}

\subsection{Mode numbers and cutoff behavior}

The stresses can shift the cutoff thickness under many stress states as shown in Figs. 4, 7 and 10. At certain core thickness, some mode may appear or disappear when stress value changes (Figs. 5, 8, and 11). The number of modes allowed in a three-layer symmetric planar waveguide is (Boyd, 1994)

$$
M \cong \frac{2 t}{\lambda} \sqrt{n^{2}-n_{1}^{2}}
$$

Stress can change the refractive index, which may change the number of modes in the waveguide. High stress value may cause multimode. Note that the cutoff behavior only exits for $m \neq 0$ modes in these plots. This is due to the symmetry of the waveguide. For the asymmetrical case, a finite mode cutoff exists even for the $m=0$ mode (Boyd, 1994).

\subsection{Loss}

When the light propagates from the unstressed area to stressed area, the refractive index and mode shape change. If the index contrast between the core and cladding regions decreases, the field confinement becomes weak. That could cause power loss (Adams, 1981; Boyd, 1994), a phenomenon referred to as radiation confinement loss. The radiation confinement loss can be obtained by calculating the ratio of power in the core to total power. Fig. 14 compares the radiation confinement loss caused by different stress states 


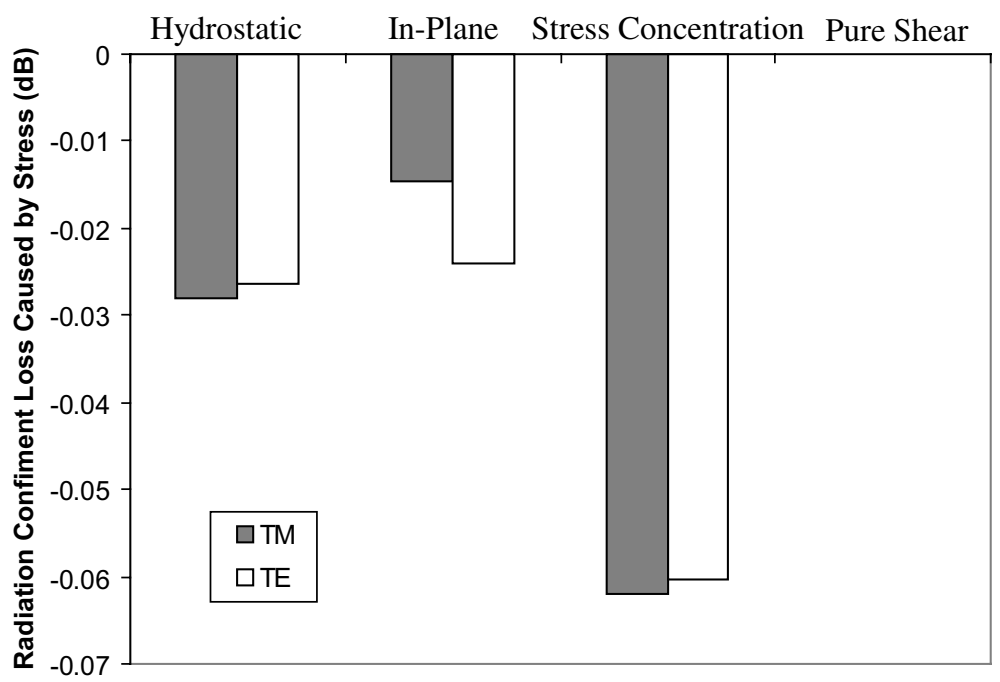

Fig. 14. Stress caused radiation confinement loss of different stress states for a symmetric planar optical waveguide with $t=1 \mu \mathrm{m}$ when $C_{1} \sigma^{*}=0.01$, where $\sigma^{*}=\sigma$ for hydrostatic and in-plane stress states; $\sigma^{*}=\sigma_{\mathrm{m}} / 10$ for stress concentration state; and $\sigma^{*}=\tau$ for pure shear state.

when $C_{1} \sigma^{*}=0.01$, where $\sigma^{*}=\sigma$ for hydrostatic and in-plane stress states; $\sigma^{*}=\sigma_{\mathrm{m}} / 10$ for stress concentration state; and $\sigma^{*}=\tau$ for pure shear state. Stress concentration causes higher confinement loss than other stress states, and the difference between TE and TM mode confinement loss is higher for in-plane stress state than for other stress states.

As the mode shape changes, the power loss may also be induced when the light transits between the unstressed and stressed areas. Transition loss can be estimated by comparing the unstressed mode shape and stressed mode shape. Fig. 15 shows the transition loss of different stress states when $C_{1} \sigma^{*}=0.01$.

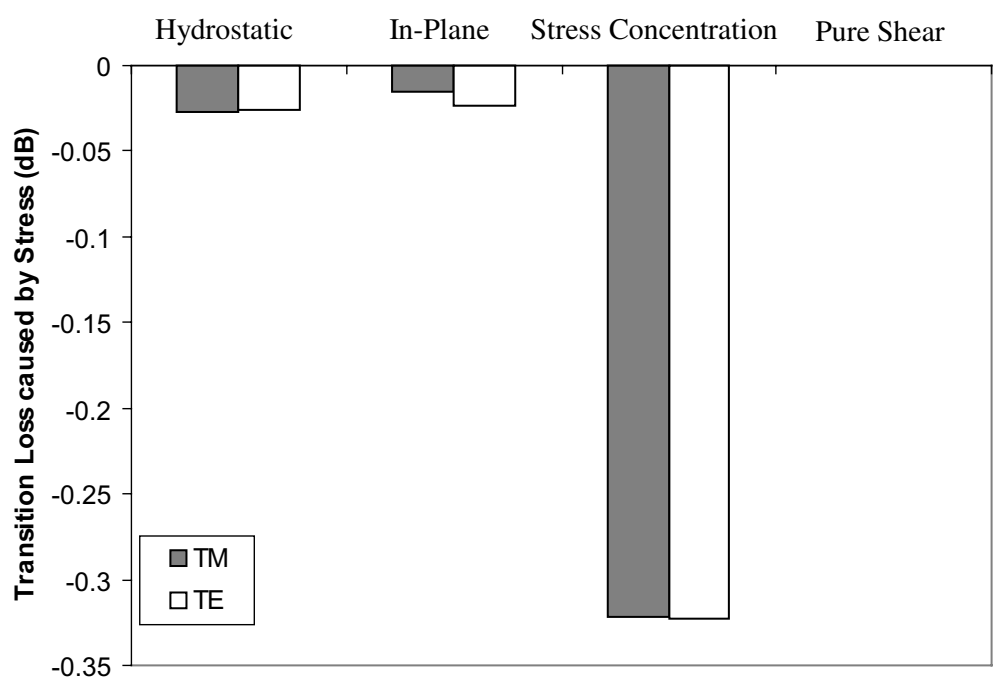

Fig. 15. Stress caused transition loss of different stress states for a symmetric planar optical waveguide with $t=1 \mu \mathrm{m}$ when $C_{1} \sigma^{*}=0.01$, where $\sigma^{*}=\sigma$ for hydrostatic and in-plane stress states; $\sigma^{*}=\sigma_{\mathrm{m}} / 10$ for stress concentration state; and $\sigma^{*}=\tau$ for pure shear state. 


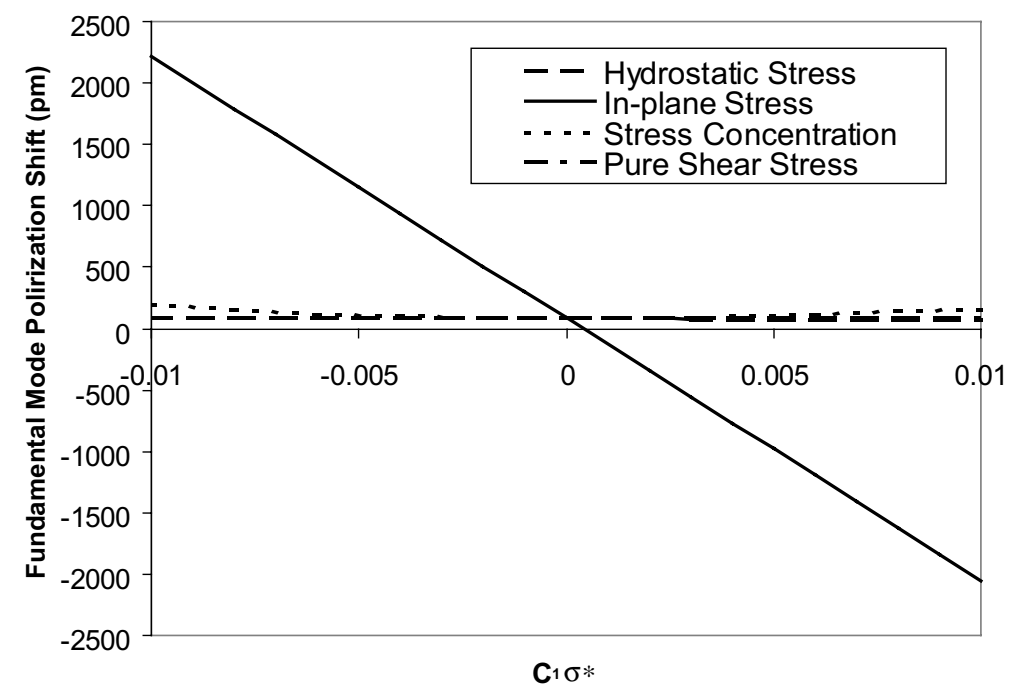

Fig. 16. Polarization shift of different stress states for a symmetric planar optical waveguide with $t=1 \mu \mathrm{m}$. In the plot, $\sigma^{*}=\sigma$ for hydrostatic and in-plane stress states; $\sigma^{*}=\sigma_{\mathrm{m}} / 10$ for stress concentration state; and $\sigma^{*}=\tau$ for pure shear state.

Because the concentrated stress significantly changes the mode shape, the transition loss caused by stress concentration is much higher than those caused by other stress states. The difference between the TE and TM mode transition loss is higher for in-plane stress state than for other stress states.

\subsection{Birefringence: polarization shift (PS) and polarization dependent loss (PDL)}

When an isotropic material is subjected to mechanical stress, it may become optically anisotropic. This phenomenon is known as birefringence (Born and Wolf, 1964). PS is used to describe the wavelength difference between the TE and TM mode, which is calculated as

$$
\mathrm{PS}=\lambda_{\mathrm{TE}}-\lambda_{\mathrm{TM}} \text {. }
$$

PS values of different stress states are shown in Fig. 16. PS values of the in-plane stress state is one order magnitude higher than those of other stress states. Stress anisotropy can dramatically increase the PS. On the other hand, PS can be controlled by applying anisotropic stresses.

In practice, the stresses are usually anisotropic and inhomogeneous as shown in Fig. 1. One of the stress components may concentrate at some area. This can cause either TM or TE mode shape to change. The transition loss of one mode becomes quit different from the other, which can induce large PDL. PDL is defined as the difference of the optical propagation losses between the TE and TM modes of a waveguide. From Figs. 14 and 15, we can see that stress anisotropy and non-uniformity can cause large PDL, which has been observed by Min et al. (2000) and Yan et al. (2002).

\section{Concluding remarks}

Modern photoelectric devices comprise many components with small feature size, complicated geometric shape and diverse materials. During processing, packaging and service, stresses are induced by many sources, such as temperature cycling (Huang et al., 2000, 2001, 2002), electron migration (Ma et al., 1995), 
oxidation (Suo, 1995), etc. The stresses can cause the refractive index change on the order of 0.01 . As shown in this paper, this small index change, aided by non-uniformity and anisotropy, causes unacceptable optical performance degradation and different stress states play different roles: high stress value may induce multimode; in-plane stresses may induce PS and PDL; stress concentration may induce large transition loss; and pure shear stress has little effects on the effective refractive index. Different waveguide structures are in different stress states. For buried waveguides, which have rectangular core shape and embedded in other cladding materials, and straight optical fibers, their stress states are similar to the hydrostatic stress state, where stresses will not cause too much loss and birefringence, but may induce multimode. For ridge waveguides and bent optical fibers, their stress states are much more complicated. Stress-induced refractive index non-uniformity and anisotropy may exist simultaneously, so many problems may be induced by stresses. Therefore, stress effects on the optical performance should be considered in the device design stage. Although this study is performed on the planar waveguides under four simple stress states, the qualitative picture obtained in this paper should be generic to other waveguide structures and under more complicated stress states.

\section{Acknowledgements}

I would like to thank Dr. X. Yan and Prof. Z. Suo for their encouragement and comments.

\section{References}

Adams, M.J., 1981. An Introduction to Optical Waveguide. John Wiley and Sons, Chichester.

Almashary, B.A., Kim, H.K., 1996. Stress-induced vertical confinement of light in bulk GaAs and Si substrates. IEEE Photonics Technology Letters 8 (10), 1358-1360.

Born, M., Wolf, W., 1964. Principal of Optics, second ed. The Macmillan Company, New York.

Boyd, J.T., 1994. Photonic integrated circuits. In: Hunsperger, R.G. (Ed.), Photonic Devices and Systems. Marcel Dekker, New York.

Buda, M., Iordache, G., Acket, G.A., van de Roer, T.G., Kaufmann, L.M.F., van Roy, B.H., Smalbrugge, E., Moerman, I., Sys, C., 2000. Stress-induced effects by the anodic oxide in ridge waveguide laser diodes. IEEE Journal of Quantum Electronics 36 (10), $1174-1183$.

Chowdhury, D., Wilcox, D., 2000. Comparison between optical fiber birefringence induced by stress anisotropy and geometric deformation. IEEE Journal of Selected Topics in Quantum Electronics 6 (2), 227-232.

Cohen, D.A., Heimbuch, M.E., Coldren, L.A., 1996. Reduced temperature sensitivity of the wavelength of a diode laser in a stressengineered hydrostatic package. Applied Physics Letters 69 (4), 455-457.

Cohen, D.A., Mason, B., Dolan, J., Burns, C., Coldren, L.A., 2000. Enhanced wavelength tuning of an InGasP-InP laser with a thermal-strain-magnifying trench. Applied Physics Letters 77 (17), 2629-2631.

Huang, M., Suo, Z., Ma, Q., 2002. Plastic ratcheting induced cracks in thin film structures. Journal of the Mechanics and Physics of Solids 50, 1079-1098.

Huang, M., Suo, Z., Ma, Q., 2001. Metal film crawling in interconnect structures caused by cyclic temperatures. Acta Materialia 49, 3039-3049.

Huang, M., Suo, Z., Ma, Q., Fujimoto, H., 2000. Thin film cracking and ratcheting caused by temperature cycling. Journal of Materials Research 15 (6), 1239-1242.

Huang, M., Yan, X., 2002. Thermal stress effects on the temperature sensitivity of optical waveguides. Journal of the Optical Society of America B, submitted for publication.

Kilian, A., Kirchhof, J., Kuhlow, B., Przyrembel, G., Wischmann, W., 2000. Birefrience free planar optical waveguide made by flame hydrolysis deposition (FHD) through tailoring of the overcladding. Journal of Lightwave Technology 18 (2), $193-198$.

Lea, E., Weiss, B.L., 1996. Photoelastic waveguides in silicon. Electronics Letters 32 (17), 1577-1579.

Maciejko, R., Berger, J., Champagne, A., Glinski, J., 1989. Biaxial stress effects on the TE/TM polarization switching of InGaAsP ridge-waveguide lasers. IEEE Photonics Technology Letters 1 (7), 162-165.

Maciejko, R., Golebiowski, A., Champagne, A., Glinski, J.M., 1993. Selected TE-TM mode pumping efficiencies for ridge-waveguide lasers in presence of stress. IEEE Journal of Quantum Electronics 29 (1), 51-61. 
Ma, Q., Chiras, S., Clarke, D.R., Suo, Z., 1995. High resolution determination of the stress in individual interconnect lines and the variation due to electromigration. Journal of Applied Physics 78, 1614-1622.

Min, Y.H., Lee, M.H., Do, J.Y., 2000. Polarization dependant loss in polymetric rib channel waveguide. IEEE Photonic Technology Letters 12 (11), 1483-1485.

Nadler, C.K., Wildermuth, E.K., Lanker, M., Hunziker, W., Melchior, H., 1999. Polarization insensitivite, low-loss, low-crosstalk wavelength multiplexer modules. IEEE Journal of Selected Topics in Quantum Electronics 5 (5), 1407-1412.

Okuno, M., Sugita, A., Jinguji, K., Kawachi, M., 1994. Birefringence control of silica waveguides on Si and its application to a polarization-beam splitter/switch. Journal of Lightwave Technology 12 (4), 625-633.

Ooba, N., Hibino, Y., Inoue, Y., Sugita, A., 2000. A thermal silica-based arrayed-waveguide grating multiplexer using bimetal plate temperature compensator. Electronics Letters 36 (21), 1800-1801.

Owyang, G.H., 1981. Foundations of Optical Waveguides. Elsevier, New York.

Rho, H., Jackson, H.E., 1999. Raman imaging of stress in a SiGe/Si photoelastic optical channel waveguide structure. Applied Physics Letters 75 (9), 1287-1289.

Saitoh, K., Koshiba, M., Tsuji, Y., 1999. Stress analysis method for elastically anisotropic material based optical waveguides and its application to strain-induced optical waveguides. Journal of Lightwave Technology 17 (2), 255-259.

Sapriel, J., 1979. Acousto-optics. John Wiley and Sons, New York (Translated by Francis, S., Kelly, B.).

Savin, S., Digonnet, M.J.F., Kino, G.S., Shaw, H.J., 2000. Tunable mechanically induced long-period fiber gratings. Optics Letters 25 (10), 710-712.

Sodha, M.S., Ghatak, A.K., 1977. Inhomogeneous Optical Waveguides. Plenum Press, New York.

Suo, Z., 1995. Wrinkling of the oxide scale on an aluminum-containing alloy at high temperature. Journal of the Mechanics and Physics of Solids 43, 829-846.

Xu, C.L., Huang, W.P., Chrostowski, J., Chaudhuri, S.K., 1994. A full-vectorial beam propagation method for anisotropic waveguides. Journal of Lightwave Technology 12 (11), 1926-1931.

Xu, J., Stroud, R., 1992. Acousto-optic Devices: Principles, Design, and Applications. John Wiley and Sons, New York.

Yan, X., Feng, D., Huang, M., 2002. Stress in cladding layers and its effect on optical performance of AWG. In: Symposium of mechanics of thin films and other small structures, 14 US National Congress of Theoretical and Applied Mechanics, Blacksburg, Virginia. 